\title{
Surgical reconstruction of peripheral pulmonary artery stenosis in Williams and Alagille syndromes
}

\author{
Michael C. Monge, MD, ${ }^{\mathrm{a}}$ Richard D. Mainwaring, MD, ${ }^{\mathrm{a}}$ Ahmad Y. Sheikh, MD, ${ }^{\mathrm{a}}$ Rajesh Punn, MD, \\ V. Mohan Reddy, MD, a and Frank L. Hanley, MD ${ }^{\mathrm{a}}$
}

\begin{abstract}
Objectives: Peripheral pulmonary artery stenosis is a rare congenital heart defect frequently found in association with Williams and Alagille syndromes. Controversy exists regarding the optimal treatment of peripheral pulmonary artery stenosis, with most centers favoring catheter-based interventions. In contrast, we have preferentially used surgical reconstruction of peripheral pulmonary artery stenosis. The purpose of the present study was to review our experience with surgical reconstruction of peripheral pulmonary artery stenosis.
\end{abstract}

\begin{abstract}
Methods: We performed a retrospective review of patients who underwent surgical reconstruction of peripheral pulmonary artery stenosis. A total of 16 patients were identified: 7 had Williams syndrome, 6 had Alagille syndrome, and 3 had no identifiable syndrome. Detailed pulmonary angiography was performed in all patients to define stenoses at the main, branch, lobar, and segmental arterial levels. The mean preoperative right ventricular/left ventricular pressure ratio was $0.88 \pm 0.07$. The surgical approach was a median sternotomy with cardiopulmonary bypass. All peripheral stenoses were augmented with pulmonary artery homograft tissue. The median age at surgery was 14 months, and concomitant procedures were performed in 9 of the 16 patients.
\end{abstract}

Results: There was 1 operative mortality $(6 \%)$. The mean right ventricular/left ventricular pressure ratio decreased to $0.40 \pm 0.04$ postoperatively $(P<.005)$, representing a 55\% reduction compared with the preoperative values. The patients were followed up for a median of 5 years. No late mortality occurred and reoperation was not required.

Conclusions: The data have demonstrate that this comprehensive surgical approach to the treatment of peripheral pulmonary artery stenosis was associated with low early and no late mortality. Surgical reconstruction of the peripheral pulmonary artery stenosis resulted in a significant decrease in right ventricular pressure. We hypothesize that this reduction in right ventricular pressures will confer a long-term survival advantage for this cohort of patients. (J Thorac Cardiovasc Surg 2013;145:476-81)

Peripheral pulmonary artery stenosis (PPAS) is a relatively rare form of congenital heart defect found in fewer than $1 \%$ of patients with congenital heart disease. Although rare, PPAS is frequently found in association with Williams and Alagille syndromes and is considered a phenotypic hallmark of these 2 genetically mediated syndromes. ${ }^{1-3}$ The pulmonary artery stenoses associated with Williams and Alagille syndromes can affect both the central and peripheral pulmonary arteries. However, it is the distal obstruction that typically has the predominant physiologic effect. The result of this obstruction is varying degrees of right ventricular (RV) hypertension,

From the Division of Pediatric Cardiac Surgery, ${ }^{\text {a }}$ Lucile Packard Children's Hospital/Stanford University School of Medicine, Stanford, Calif; and Division of Pediatric Cardiology, ${ }^{\mathrm{b}}$ Lucile Packard Children's Hospital/Stanford University School of Medicine, Stanford, Calif.

Disclosures: Authors have nothing to disclose with regard to commercial support.

Read at the 38th Annual Meeting of The Western Thoracic Surgical Association, Maui, Hawaii, June 27-30, 2012.

Received for publication June 17, 2012; revisions received Aug 16, 2012; accepted for publication Sept 20, 2012; available ahead of print Dec 10, 2012.

Address for reprints: Richard D. Mainwaring, MD, Division of Pediatric Cardiac Surgery, Lucile Packard Children's Hospital/Stanford University School of Medicine, 300 Pasteur Dr, Falk CVRC, Stanford, CA 94305 (E-mail: mainwaring@stanford. edu).

0022-5223/\$36.00

Copyright (c) 2013 by The American Association for Thoracic Surgery

http://dx.doi.org/10.1016/j.jtcvs.2012.09.102 which can be graded from mild to moderate to severe. The adverse physiologic consequences of RV hypertension have been well documented and include hypertrophy, systolic and diastolic dysfunction, and, eventually, can lead to ventricular failure in some cases. ${ }^{4,5}$

Considerable debate has occurred in published studies regarding the natural history of PPAS. Two sources of evidence have suggested that PPAS associated with Williams syndrome might undergo a process of spontaneous regression. Stamm and colleagues ${ }^{6}$ noted an inverse correlation between patient age at presentation and the degree of RV hypertension. These data have been interpreted to indicate that $\mathrm{RV}$ hypertension decreases over time. In addition, several small series have demonstrated a decrease in RV pressures in patients evaluated by serial cardiac catheterization. ${ }^{7-10}$ Most patients who did demonstrate spontaneous regression had mild to moderate PPAS, and spontaneous regression of severe PPAS has been unusual enough to warrant a case report. ${ }^{11}$ Currently, no evidence has indicated that PPAS associated with the Alagille syndrome can undergo this same process of spontaneous regression.

It is not surprising that the optimal management algorithm for treating PPAS has yet to be established, with multiple reasons for this ambiguity. First, PPAS and the 2 


$\begin{array}{ll}\text { Abbreviations and Acronyms } \\ \text { PA/VSD/ } & =\text { pulmonary atresia/ventricular septal } \\ \text { MAPCAs } & \text { defect/major aortopulmonary } \\ & \text { collateral arteries } \\ \text { PPAS } & =\text { peripheral pulmonary artery stenosis } \\ \text { LV } & =\text { left ventricular } \\ \text { RV } & =\text { right ventricular }\end{array}$

genetic syndromes most closely associated with this condition are relatively rare. Second, the possibility that some patients with PPAS will demonstrate spontaneous regression has ameliorated the perceived mandate for intervention. Finally, surgical reconstruction of the lobar and segmental pulmonary arteries has not been a standard part of the armamentarium for most congenital heart centers, rendering PPAS to a nonoperative treatment option in most circumstances.

Most patients with Williams syndrome have supravalvar aortic stenosis, a subject that has garnered an enormous amount of attention during the past several decades. Dozens of reports have focused on the surgical management of supravalvar aortic stenosis, debating the merits of single, dual, or 3-sinus repair. ${ }^{12-14}$ In contrast, very limited information exists on the treatment of patients with both systemic and pulmonary outflow tract obstruction, ${ }^{6,15}$ with most centers advocating a "multimodality approach." This strategy includes catheter-based interventions to address the PPAS ${ }^{16,17}$ and reserving surgery to repair the supravalvar aortic stenosis and central pulmonary arterial stenoses.

Our center has acquired extensive experience in the treatment of pulmonary atresia with a ventricular septal defect and major aortopulmonary collateral arteries (PA/VSD/ MAPCAs). This work frequently requires reconstruction of PPASs at the segmental and lobar levels. Because of this significant surgical experience with peripheral vessel reconstruction in PA/VSD/MAPCAs, we have applied these same techniques to the reconstruction of PPASs associated with Williams and Alagille syndromes. The present report summarizes the surgical approach and the results of our surgery-based approach to PPAS.

\section{MATERIALS AND METHODS}

The institutional review board at Stanford University approved the study protocol. Patients undergoing surgical reconstruction of the PPAS were identified from the cardiac database. A written questionnaire and Health Insurance Portability and Accountability Act authorization form were sent to the families. A returned questionnaire signified consent by the parents for a review of the medical records. A review of the medical records, along with the questionnaire, provided the basis for ascertaining the current health status of the children.

The present study summarizes our surgical experience from 2002 to 2012 with 16 consecutive patients who had PPAS with severe RV hypertension. We defined "severe" RV pressures as RV pressures that exceeded two thirds of the systemic pressure. Of the 16 patients, 7 had Williams syndrome, 6 had Alagille syndrome, and 3 had no identifiable syndrome; 9 were female and 7 were male. The median age at surgery was 14 months (range, 2 months to 13 years), and the median weight at surgery was 8.7 $\mathrm{kg}$ (range, 3.3 to $28.5 \mathrm{~kg}$ ). Six patients underwent preoperative balloon dilation of the PPAS, with an average decrease in RV pressure of $10 \mathrm{~mm} \mathrm{Hg}$.

All 16 patients underwent cardiac catheterization for evaluation of the hemodynamics and to provide a "roadmap" for reconstruction of the pulmonary arterial system. The mean RV/left ventricular (LV) pressure ratio was $0.88 \pm 0.07$ (range, 0.72-1.00). For the patients with Williams syndrome who had supravalvar aortic stenosis, the descending thoracic aortic pressure measurement was substituted for the LV pressure. The pulmonary angiogram (Figure 1, A) demonstrated diminutive, but confluent, central branch pulmonary arteries. The ostial stenoses of the right upper lobe segments can be faintly seen. Separate right (Figure 1,B) and left (Figure 1,C) pulmonary angiograms highlighted the multiple lobar and segmental branch stenoses affecting the right upper, middle, and lower lobes and left upper lobe. This preoperative anatomy has been recapitulated in an artist's illustration (Figure 2, $A$ ).

Surgical reconstruction was performed by way of a median sternotomy and used cardiopulmonary bypass support. All stenoses identified by angiography were augmented using pulmonary artery homograft tissue. The surgical technique we use includes external dissection of the affected arterial branch to obtain distal vascular control. This dissection can lead to the edge of the lung parenchyma, which can be safely brushed back to achieve distal access for reconstruction of the lobar and segmental branches. The entirety of this dissection is performed with electrocautery and before heparinization, because dissection near the lung tissue after heparinization can result in significant intraparenchymal hemorrhage. After cardiopulmonary bypass has been established, both sides of the heart are decompressed (a LV vent is inserted through the right superior pulmonary vein), which greatly facilitates surgical exposure. The affected arteries are incised longitudinally through the area of stenosis and onto the normal distal vessel for at least $1 \mathrm{~cm}$ beyond the endothelial abnormality (Figure 2, B). The median number of reconstructed peripheral arterial stenoses was 7 (range, 5-10). The main pulmonary artery and central branch pulmonary arteries were enlarged to achieve a normal size for the patients' body surface area (Figure 2, C).

Of the 16 patients, 9 had associated cardiac anomalies (Table 1). Of the 7 patients with Williams syndrome, 6 had supravalvar aortic stenosis. The median LV outflow tract gradient was $65 \mathrm{~mm} \mathrm{Hg}$ (range, $45-100 \mathrm{~mm} \mathrm{Hg}$ ). Aortic cross-clamping was performed only to address these associated cardiac lesions.

Statistical analysis was performed using a paired $t$ test.

\section{RESULTS}

The 16 patients in the present series underwent 17 surgical reconstructions of the PPAS, with 1 operative mortality. The sole death occurred in 1 patient with Williams syndrome. That patient had concomitant repair of supra-aortic valvar stenosis and PPAS and postoperatively demonstrated profound LV dysfunction, progressed to multisystem organ failure, and subsequently died. The median cardiopulmonary bypass time for the entire group was 209 minutes (range, 78-312 minutes), and the median cross- clamp time for the 9 patients with associated cardiac malformations was 35 minutes (range, 7-51 minutes). The median duration hospital stay for the 15 survivors was 14 days (range, 8-40 days).

The RV/LV pressure ratios decreased from $0.88 \pm 0.07$ preoperatively to $0.40 \pm 0.04$ (range, $0.24-0.54$ ) in the immediate postoperative period, representing a $55 \%$ reduction 

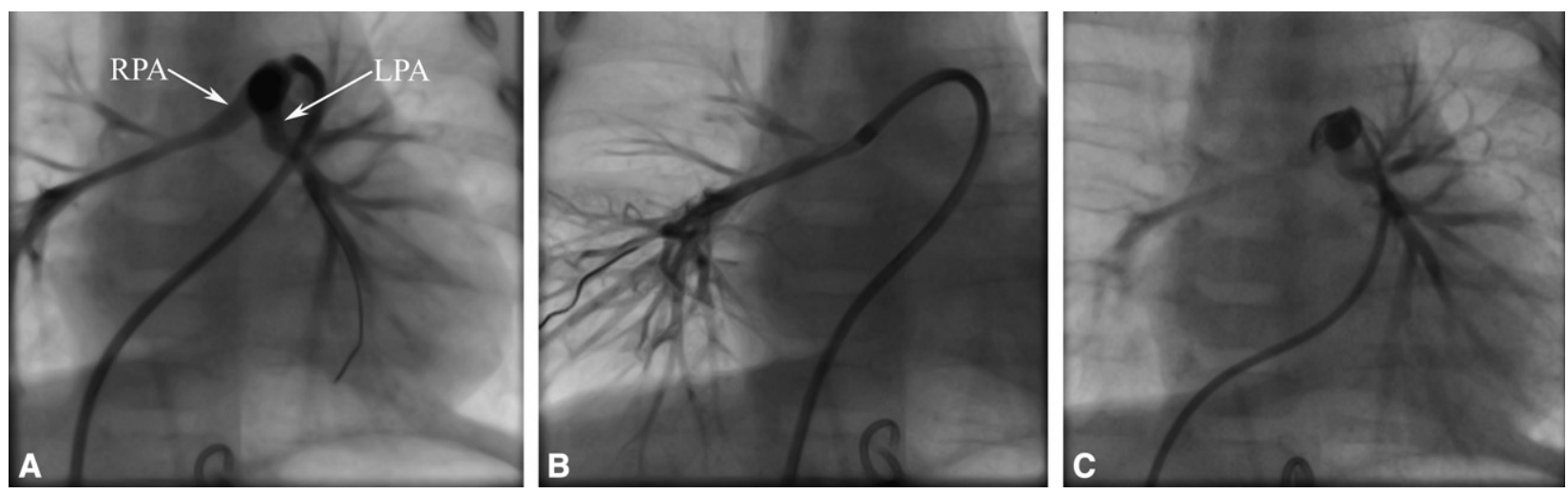

FIGURE 1. A, Pulmonary angiogram demonstrating diminutive but confluent central branch pulmonary arteries. Ostial stenoses are evident at 2 branches to right upper lobe. Separate $\mathrm{B}$, right and $\mathrm{C}$, left pulmonary angiograms demonstrating multiple stenoses involving right upper, middle, and lower lobes and left upper lobe. $R P A$, Right pulmonary artery; $L P A$, left pulmonary artery.

in the $\mathrm{RV} / \mathrm{LV}$ pressure ratio $(P<.005)$. Late hemodynamics were assessed at $4.1 \pm 1.1$ years postoperatively, with an average $\mathrm{RV} / \mathrm{LV}$ pressure ratio of $0.38 \pm 0.04(P<.005$ compared with preoperatively). These data are summarized in Figure 3.

The pre- and postoperative hemodynamics for the 3 subgroups of patients (ie, Williams syndrome, Alagille syndrome, and no identifiable syndrome) are shown in Figure 4. No discernible difference was found among these 3 groups of patients, with the caveat that the number of patients in each category was quite small.

The follow-up duration for the 15 survivors was $5 \pm 2$ years (range, 4 months to 10 years). The questionnaires from the families provided up-to-date documentation of the health status for the 15 survivors, including those patients who were referred to our center for surgery and have been followed up by their outside institutions. No late mortality occurred, and none of the patients required surgical reoperation. A single patient underwent balloon dilation for a residual PPAS. That patient had the greatest RV/LV pressure ratio (0.54) postoperatively and subsequently had abnormal lung perfusion scan findings, prompting reinvestigation with cardiac catheterization. The remaining 14 patients were followed up clinically and using echocardiography to assess the $\mathrm{RV}$ pressures.

\section{DISCUSSION}

The present study summarizes our institutional experience with comprehensive surgical reconstruction of PPAS in 16 consecutive patients with severe RV hypertension. This approach was associated with low early $(6.3 \%)$ and no late mortality. Surgical reconstruction resulted in an average $55 \%$ reduction in the RV/aortic pressure ratio. This decrease was maintained during the 4 years of hemodynamic follow-up. These results have indicated that surgical reconstruction of the PPAS might be superior to the conventional approach using catheter-based interventions.
Our surgical group has acquired extensive experience with the treatment of patients with PA/VSD/MAPCAs. The surgical techniques developed for unifocalization of MAPCAs frequently requires extensive reconstruction of diminutive vessels well beyond the hilum of the lung. Experience with more than 700 patients has emphasized the importance of incorporating as many unobstructed pulmonary segments as possible. ${ }^{18}$ A survival advantage for those patients who achieve complete intracardiac repair has been documented and has been further accentuated in those with the lowest RV pressure. We recently published our experience with 15 patients who had Alagille syndrome in association with PA/VSD/MAPCAs. ${ }^{19}$ That report demonstrated that the unifocalization approach can be successfully used in patients with Alagille syndrome despite the existence of published data suggesting that syndromic patients might be at considerably greater risk than their nonsyndromic counterparts. ${ }^{20-22}$

From the surgical techniques developed for PA/VSD/ MAPCAs, we have adopted a surgical approach to the management of PPAS. Of the present 16 patients, 15 underwent single-stage reconstruction, and 9 of these patients also underwent concomitant procedures. In a single patient with Alagille syndrome, 2-stage reconstruction was performed. That patient had a marked disparity in blood flow to the right $(85 \%)$ and left (15\%) lungs owing to a tight ostial stenosis of the left branch pulmonary artery. It was our preconception that complete bilateral repair might result in persistence of this disparity in blood flow and might also risk reperfusion injury. We consequently planned a 2 -stage approach, with the initial operation addressing the left branch pulmonary artery stenosis. The patient underwent a second operation 6 months later, at which time 7 lobar or segmental stenoses were repaired on the right. This specific case exemplifies the considerable diversity in the anatomy that can be encountered in this relatively small study group. 

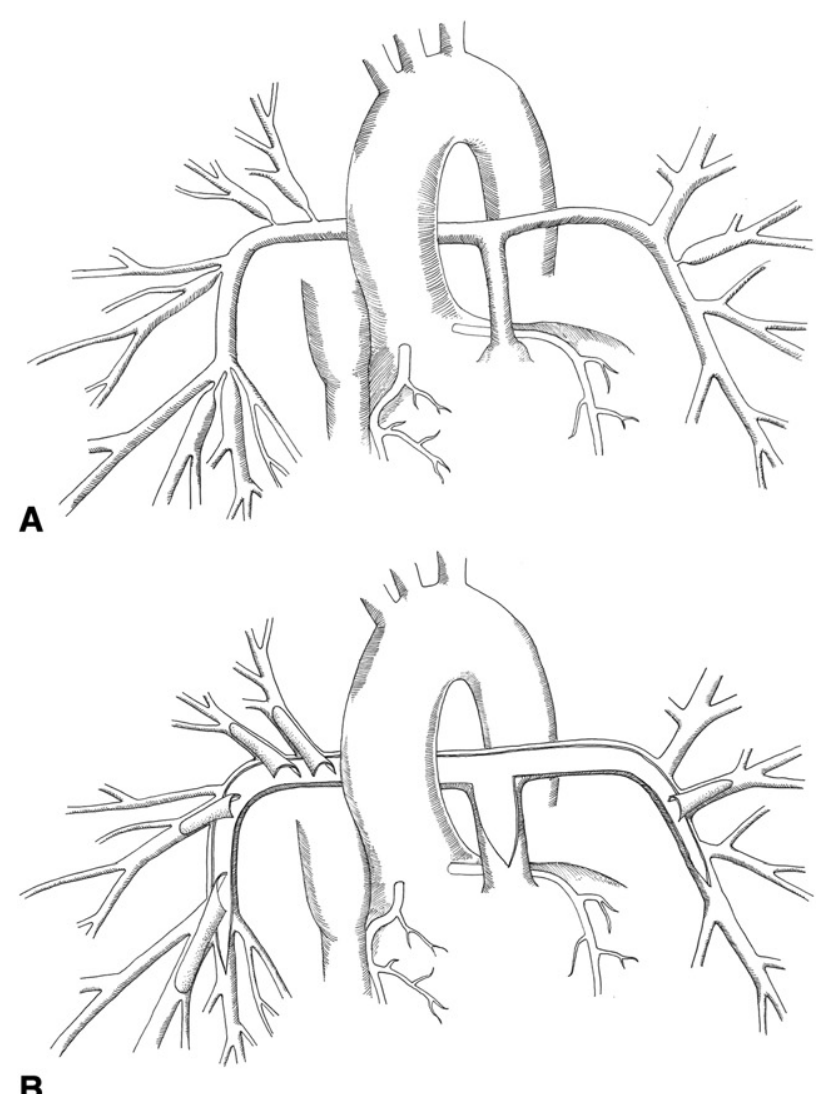

B

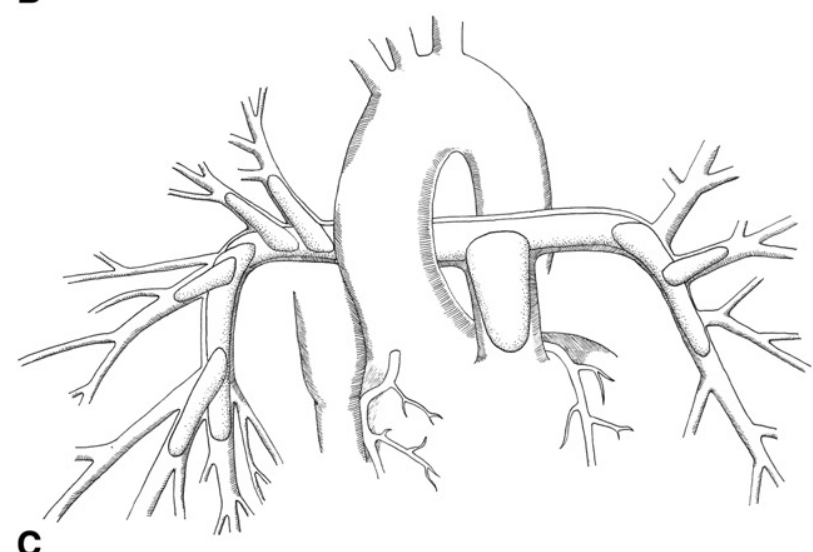

FIGURE 2. A, Artist rendition of pulmonary artery anatomy recapitulating findings from pulmonary angiogram and showing 6 stenotic lobar or segmental branches affected. B, Artist illustration of surgical technique for reconstruction of multiple peripheral pulmonary artery stenoses. Each affected lobar or segmental branch is incised and then augmented with a patch of pulmonary artery homograft tissue. C, Artist illustration of surgical reconstruction of main pulmonary artery, central branch pulmonary arteries, and distal branch pulmonary arteries using 4 separate homograft patches. The goal is to achieve a normal cross-sectional diameter according to the body surface area of the patient. Illustrations by Erin Anne Mainwaring.

We included 3 patients in the present series who did not have Williams or Alagille syndrome but otherwise shared the cardiac phenotype of PPAS with severe RV
TABLE 1. Concomitant procedures performed at PPAS reconstruction

\begin{tabular}{ll}
\hline \multicolumn{1}{c}{ Concomitant procedure } & $\mathbf{n}$ \\
\hline Repair of supravalvar aortic stenosis & 6 \\
Repair of atrial septal defect & 3 \\
Repair of ventricular septal defect & 2 \\
Repair of left main coronary artery stenosis & 1 \\
Repair of subaortic stenosis & 1 \\
\hline
\end{tabular}

PPAS, Peripheral pulmonary artery stenosis.

hypertension. These 3 patients had a preoperative RV/LV pressure ratio of $0.92,0.89$, and 0.83 . Of these 3 patients, 1 also had a critical stenosis of the proximal left main coronary artery and moderate narrowing of the innominate and left carotid arteries. These associated findings would be most typical of patients with Williams syndrome. We could not discern any anatomic features that could distinguish among the 3 subgroups of patients (Williams syndrome, Alagille syndrome, or those without an identifiable syndrome) in our study.

PPAS has a wide variety of anatomic manifestations. Most patients will have relatively small main and central branch pulmonary arteries, even though these vessels conduct a full cardiac output. The diminutive size of the branch pulmonary arteries typically extends into each hilum. Invariably, multiple stenoses of the lobar and segmental branches are also present giving rise to the major portion of the RV hypertension. We have observed many different types of stenoses in these smaller caliber vessels. Some stenoses are discrete and located at the ostia, some are discrete stenoses in the midvessel, and other stenoses can be long and tubular. Surgical repair using homograft patch augmentation is applicable to all types of stenoses and effectively relieves the obstruction. The physiology of the patients before surgery and after repair did not seem to be influenced by the presence of Williams syndrome, Alagille syndrome, or the absence of an identifiable syndrome. This was illustrated in Figure 4, demonstrating comparable RV/aortic

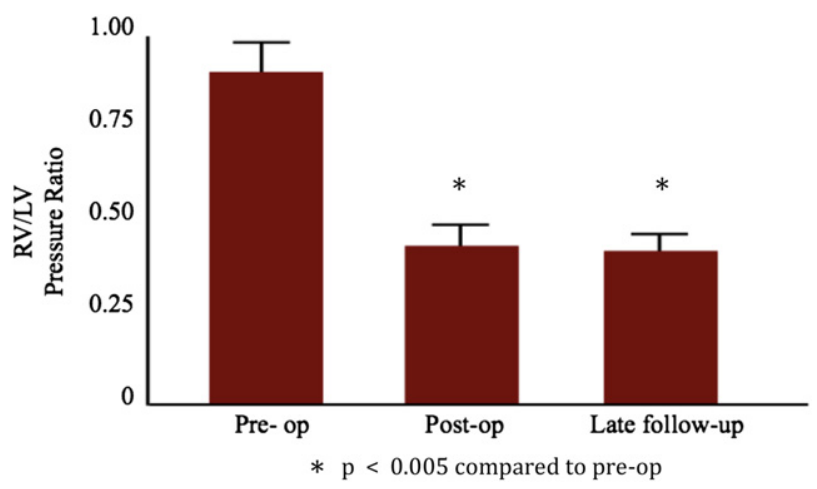

FIGURE 3. Bar graph demonstrating comparison of pre- and postoperative right ventricular/left ventricular $(R V / L V)$ pressure ratios. Late assessment of hemodynamics performed an average of 4 years after surgery. 


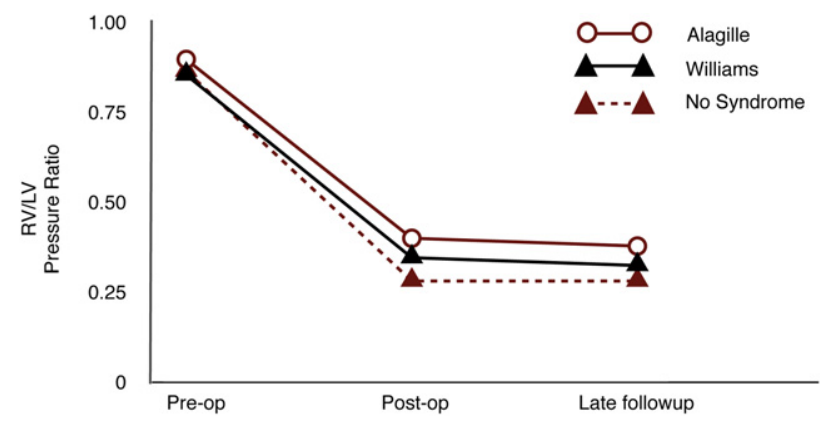

FIGURE 4. Line graph showing pre- and postoperative hemodynamics for 3 subsets of patients (Williams, Alagille, and no identifiable syndrome). $R V / L V$, Right ventricular/left ventricular.

pressures ratios in all 3 cohorts both before and after surgery.

A modest amount of published data are available on interventional cardiology techniques for the management of PPAS associated with the Williams and Alagille syndromes. ${ }^{16,17}$ The indications for intervention have been defined rather strictly systemic or suprasystemic RV pressures. Patients with such RV pressures have the greatest physiologic burden, the greatest risk of sudden death, ${ }^{23}$ and the lowest possibility of experiencing spontaneous regression. Geggel and colleagues ${ }^{17}$ summarized their experience with 25 patients undergoing balloon dilation of severe PPAS in association with Williams syndrome. That intervention resulted in a significant increase in the pulmonary artery diameter, but the RV pressures were unchanged after dilation. Thus, the physiologic benefit of this approach remains questionable in the very cohort for whom spontaneous regression is the least likely. Less experience is available regarding the efficacy of balloon dilation of PPAS in patients with Alagille syndrome. It has been postulated that the stenoses associated with the Williams and Alagille syndromes are at high risk of balloon complications, including perforation, dissection, and aneurysm formation. ${ }^{15}$

In our series, 6 patients had undergone balloon dilation of PPAS before the surgical referral. Of these 6 patients, 3 had undergone this procedure at outside institutions. All 3 patients who had undergone balloon dilation at our institution had do so in the earlier portion of our experience, and 1 of these procedures precipitated a surgical emergency secondary to rupture of a lobar branch pulmonary artery. The results of these attempted dilation procedures recapitulate the experience highlighted in the previous paragraph, with minimal physiologic benefit and 1 serious complication. PPAS is invariably diagnosed by cardiologists and other specialists who might make decisions about care before the surgical consultation, a situation paralleled in many other areas of medicine. Our current preference and recommendation would be to perform diagnostic cardiac catheterization, followed by primary surgical reconstruction of all patients with PPAS associated with severe RV hypertension.
The surgical data on the subject of PPAS are quite limited and have uniformly advocated a "multimodality approach." 6,12,15 This strategy uses catheter-based interventions to address PPAS, followed by surgery, for the treatment of the remainder of the heart defects (usually supravalvar aortic stenosis). The rationale put forth to justify this approach is that preoperative balloon dilation could potentially decrease the RV pressure and thereby reduce the risk of the subsequent surgical procedure. ${ }^{23}$ In a series of 33 surgical patients with Williams syndrome reported by Stamm and colleauges, ${ }^{6} 18$ patients had a significant enough PPAS to warrant intervention. Of these 18 patients, 8 underwent preoperative balloon dilation, 3 of whom subsequently did not require surgery for PPAS. The remaining 15 patients underwent surgery of their pulmonary arteries, largely confined to reconstruction of the main and central branch pulmonary arteries. There were 3 operative deaths $(20 \%)$, and the median postoperative RV/aortic pressure ratio was 0.54 . Furthermore, 8 of the 12 long-term survivors $(67 \%)$ required repeat intervention on their pulmonary arteries. Thus, this multimodality approach resulted in relatively high RV/aortic pressure ratios and a significant incidence of reintervention.

The present study had several limitations. First, we were not able to estimate the true number of patients born with PPAS and severe RV hypertension. Our surgical program serves as a referral center for patients with PA/VSD/MAPCAs, and we also receive referrals for PPAS from a wide geographical area. However, in that referral area, numerous possibilities are available for patients with PPAS. Some patients could experience spontaneous regression, although this seems unlikely in the group of patients with severe $\mathrm{RV}$ hypertension. Some patients could die before diagnosis or treatment, and others might not be treated because of the complexity of the condition and ambiguity of the existing data. It is also possible that some patients underwent successful catheter-based treatment, or conversely, could have sustained a fatal complication from that intervention. A second limitation of the present study was that we could not estimate the number of patients with milder forms of PPAS (ie, patients with RV/LV pressure ratios less than two thirds). These patients are much more likely to experience spontaneous resolution and are also much more likely to respond favorably to balloon dilation. Thus, although we have provided data and commentary on the 16 consecutive patients who were referred for surgical treatment of PPAS and severe RV hypertension, we could not provide an estimate for the denominator of this entity or the incidence of severe RV hypertension compared with the milder forms of PPAS.

\section{CONCLUSIONS}

We have summarized our data from a series of 16 patients with PPAS who underwent surgical reconstruction as the 
primary mode of treatment. This approach represents a significant divergence from that currently advocated in published reports. We believe the results of the present study vindicate a surgery-based approach to PPAS, because the surgical mortality rate, postoperative RV/aortic pressure ratios, and reintervention rates compared quite favorably to those achieved using the conventional multimodality approach. We hypothesize that the reduction in RV pressure will confer a long-term survival advantage and improve patients' overall quality of life.

\section{References}

1. Williams JC, Barratt-Boyes BG, Lowe JB. Supravalvular aortic stenosis. Circulation. 1961;24:1311-8.

2. Beuren AJ, Schulze C, Eberle P, Harmjanz D, Apitz J. The syndrome of supravalvular aortic stenosis, peripheral pulmonary stenosis, mental retardation and similar facial appearance. Am J Cardiol. 1964;13:471-83.

3. McElhinney DB, Krantz ID, Bason L, Piccoli DA, Emerick KM, Spinner NB, et al. Analysis of cardiovascular phenotype and genotype-phenotype correlation in individuals with a JAG1 mutation and/or Alagille syndrome. Circulation. 2002; $106: 2567-74$

4. Katz NM, Blackstone EH, Kirklin JW, Pacifico AD, Bargeron LM Jr. Late survival and symptoms after repair of tetralogy of Fallot. Circulation. 1982;62:403-10.

5. Kirklin JW, Blackstone EH, Jonas RA, Shimazaki Y, Kirklin JK, Mayer JE Jr, et al. Morphologic and surgical determinants of outcome events after repair of tetralogy of Fallot and pulmonary stenosis: a two-institution study. J Thorac Cardiovasc Surg. 1992;103:706-23.

6. Stamm C, Friehs I, Moran AM, Zurakowski D, Bacha E, Mayer JE, et al. Surgery for bilateral outflow tract obstruction in elastin arteriopathy. J Thorac Cardiovasc Surg. 2000;120:755-63.

7. Giddins NG, Finley JP, Nanton MA, Roy DL. The natural course of supravalvar aortic stenosis and peripheral pulmonary artery stenosis in Williams syndrome. Br Heart J. 1989;62:315-9.

8. Wren C, Oslizlok P, Bull C. Natural history of supravalvular aortic stenosis and pulmonary artery stenosis. JACC. 1990;15:1625-30.

9. Wessel A, Pankau R, Kececioglu D, Ruschewski W, Bursch JH. Three decades of follow-up of aortic and pulmonary vascular lesions in the Williams-Beuren syndrome. Am J Med Genet. 1994;52:297-301.
10. Kim YM, Yoo SJ, Choi JY, Kim SH, Bae EJ, Lee YT. Natural course of supravalvar aortic stenosis and peripheral pulmonary arterial stenosis in Williams syndrome. Cardiol Young. 1999;9:37-41.

11. Miyamura H, Watanabe H, Tatebe S, Eguchi S. Spontaneous regression of peripheral pulmonary artery stenosis in Williams syndrome. Jpn Circ J. 1996;60: 311-4.

12. Hickey EJ, Jung G, Williams WG, Manlhiot C, Van Arsdell GS, Caldarone CA, et al. Congenital supravalvular aortic stenosis: defining surgical and nonsurgical outcomes. Ann Thorac Surg. 2008;86:1919-27.

13. Stamm C, Kreutzer C, Zurakowski D, Nollert G, Friehs I, Mayer JE, et al. Fortyone years of surgical experience with congenital supravalvular aortic stenosis J Thorac Cardiovasc Surg. 1999;118:874-85.

14. Kaushal S, Backer CL, Patel S, Gossett JG, Mavroudis C. Mid-term outcomes in supravalvular aortic stenosis demonstrate the superiority of multisinus aortoplasty. Ann Thorac Surg. 2010;89:1371-7.

15. Gandy KL, Tweddell JS, Petech AN. How we approach peripheral pulmonary stenosis in Williams-Beuren syndrome. Semin Thorac Cardiovasc Surg Pediatr Card Surg Ann. 2009;12:118-21.

16. Trivedi KR, Benson LN. Interventional strategies in the management of peripheral pulmonary artery stenosis. J Interven Cardiol. 2003;16:171-88.

17. Geggel RL, Gauvreau K, Lock JE. Balloon dilation angioplasty of peripheral pulmonary stenosis associated with Williams syndrome. Circulation. 2001;103: 2165-70.

18. Malhotra S, Hanley FL. Surgical management of pulmonary atresia with ventricular septal defect and major aortopulmonary collaterals: a protocol-based approach. Semin Thorac Cardiovasc Surg Pediatr Card Surg Ann. 2009;12: 145-51.

19. Mainwaring RD, Sheikh AY, Punn R, Reddy VM, Hanley FL. Surgical outcomes for patients with pulmonary atresia/major aortopulmonary collaterals and Alagille syndrome. Eur J Cardiothorac Surg. 2012;42:235-40.

20. Formigari R, Michielon G, Digillio MC, Piacentini G, Carotti A, Giardini A, et al Genetic syndromes and congenital heart defects: how is surgical management affected? Eur J Cardiothorac Surg. 2009;35:606-14.

21. Michielon G, Marino B, Formigari R, Gargiulo G, Picchio F, Digilio MC, et al. Genetic syndromes and outcome after surgical correction of tetralogy of Fallot. Ann Thorac Surg. 2006;81:968-75.

22. Blue GM, Mah JM, Cole AD, Lal V, Wilson MJ, Chard RB, et al. The negative impact of Alagille syndrome on survival of infants with pulmonary atresia. J Thorac Cardiovasc Surg. 2007;133:1094-6.

23. Burch TM, McGowan FX, Kussman BD, Powell AJ, DiNardo JA. Congenital supravalvular aortic stenosis and sudden death associated with anesthesia: what's the mystery? Anesthesia. 2008;107:1848-54. 